\section{Imported bancroftian filariasis discovered in a patient infected with Plasmodium falciparum: First case of concomitant parasitism in the Al-Buraimi Governorate, Oman}

\author{
Muhammad Muqeet Ullah, Ahmed Yar \\ Mohammed Dawood Al Balushi, Nader \\ Rahid Salim Al Aliyani, Biju Kalarikkal, \\ Reeher Ian C. Miranda, Sherif \\ Mohamed Sherif, Ahmed Salim \\ Mubarak Al Habsi, Eman Khalfan Al \\ Saidi, Adil Anver, Nouraldeen Hasan \\ Qassem
}

Directorate of Disease Surveillance and Control, Directorate General of Health Services, Al Buraimi Governorate, Ministry of Health, Sultanate of Oman

\begin{abstract}
Malaria and lymphatic filariasis (LF) are endemic in tropical and subtropical regions of sub-Saharan Africa, Asia, and Central and South America. Ninety-two percent (200 million) of malaria cases in 2017 were detected in the WHO African Region. This accounts for approximately $30 \%$ of the global burden of LF disease and includes 405.9 million people at risk in 39 out of 46 member countries. Anopheles species of mosquitoes transmit Wuchereria bancrofti in most parts of Africa. Our case of a 23-year-old Nigerian woman highlights incidental laboratory findings showing the first malaria/filariasis coinfection in the governorate (province). This coinfection was ascertained during the usual medical screening before recruitment in Oman, which is routinely conducted for every expatriate.
\end{abstract}

\section{Introduction}

Globally, it is estimated that in 2017 there were 219 million cases of malaria. WHO African Region accounted for 200 million ( $92 \%$ ) cases of malaria, followed by the WHO South-East Asia Region (5\%) and the WHO Eastern Mediterranean Region (2\%). Five countries: Nigeria (25\%), Democratic Republic of the Congo (11\%), Mozambique (5\%), India (4\%) and Uganda (4\%) accounted for nearly half of all malaria cases worldwide. In 2017, the WHO African Region accounted for $99.7 \%$ of estimated malaria cases due to Plasmodium falciparum, as well as the WHO regions of
South-East Asia (62.8\%), Eastern Mediterranean (69\%) and Western Pacific $(71.9 \%) .{ }^{1}$ In the past, Malaria was one of the major public health problems in Oman. Data analysis showed that the main sources of malaria infestation into Oman came from East Africa and Indian subcontinent. Consequently, appropriate strategies were implemented in Oman, including the distribution of chemoprophylaxis for travelers and the screening of expatriates arriving from endemic countries at primary health care and Medical Fitness Examination Centers, at a governorate (provincial) level.

It is estimated that about 120 million people from 81 countries have been infected with lymphatic filariasis. Approximately, about $65 \%$ of people at risk reside in WHO South-East Asia Region, 30\% in the African Region and the rest in the other parts of the tropical world. ${ }^{2}$ Anopheles mosquitoes species transmit Wuchereria bancrofti in most parts of Africa and W. bancrofti is responsible for $90 \%$ of cases. ${ }^{3}$ Lymphatic filariasis, commonly known as elephantiasis, is a neglected tropical disease. The painful and deeply disfiguring visible manifestations of the disease such as lymphoedema, elephantiasis and scrotal swelling occur later in life and can lead to permanent disabilities.

Lymphatic filariasis (LF) is transmitted by different types of mosquitoes, e.g. the Culex mosquito, which is widespread in urban and semi-urban areas; Anopheles, mainly found in rural areas; or Aedes, mainly in endemic islands in the Pacific.

LF is a vector-borne disease caused by nematode parasites of the Filarioidea family, namely Wuchereria bancrofti (Cobbold, 1977), Brugia malayi (Brug, 1927), and Brugia timori (Partona et al., 1977). ${ }^{4}$

Oman is considered to be free from LF, with only sporadic cases coming from endemic areas. However, vectors such as Anopheles subtypes and Culicine mosquitoes including Culex are endemic in some parts of Oman, However, thanks to a strong and vigilant surveillance and control system, no indigenous or local case of LF were so far reported.

\section{Case Report}

In June 2019, a 23-year-old female housemaid underwent screening at the Medical Fitness and Examination Center in the Al Buraimi Governorate (Province) in Oman, after arriving from her home country Nigeria [endemic for filariasis and malaria under Mass Drug Administration (MDA) strategy for filariasis by the WHO].
Correspondence: Muhammad Muqeet Ullah, Regional Epidemiologist, Directorate of Disease Surveillance and Control, Directorate General of Health Services, Al Buraimi Governorate, Ministry of Health, Sultanate of Oman. E-mail: drmuquetullah@yahoo.com

Key words: Lymphatic Filariasis, Malaria, Wuchereria Bancrofti.

Acknowledgments: The authors are grateful to the medical technologists of the Medical Fitness and Examination Center laboratory and Al Buraimi Hospital laboratory for detection of Microfilaria as well as medical team from Al-Buraimi Hospital for management support. We appreciated the efforts of Public health team for screening and follow up of patient for completion of treatment.

Contributions: The authors contributed equally.

Conflict of interest: The authors declare no potential conflict of interest.

Funding: None.

Ethics approval: Official approval from $\mathrm{Al}$ Buraimi Hospital Director and Provincial Director General was taken formally as requirement from Regional Research and Ethical Committee, Al Buraimi Governorate, Oman.

Informed consent: Formal informed consent from the patient was taken verbally as well as in appropriate form. Personal information including name and initials will not be published and efforts will be made to conceal identity as much as possible but only clinical details and microscopic images to be reported and published but anonymity cannot be guaranteed.

Received for publication: 11 September 2019 Revision received: 18 January 2020.

Accepted for publication: 28 February 2020.

This work is licensed under a Creative Commons Attribution-NonCommercial 4.0 International License (CC BY-NC 4.0).

OCopyright: the Author(s), 2020

Licensee PAGEPress, Italy

Infectious Disease Reports 2020; 12:8304

doi:10.4081/idr.2020.8304

\section{Onset of symptoms}

The patient presented with a mild fever and headache from 2 days before arriving in Oman. She had left lower limb mild intermittent pain since few months. The case was referred to Al Buraimi regional hospital for management after diagnosis on peripheral smear. The patient had previous history of malaria in the last months of 2018. 


\section{General examination}

The patient was afebrile $\left(37^{\circ} \mathrm{C}\right)$, her blood pressure was $135 / 75 \mathrm{mmHg}$ ), pulse 70/min, she was conscious and oriented.

\section{Laboratory findings}

Full blood count showed: hemoglobin $12.38 \mathrm{~g} / \mathrm{dl}$, white blood cells (WBC) count 6000 per ul, red blood cell (RBC) count 4.58 million per $\mathrm{ul}$ and platelets 29600 per ul, within normal range. Glucose-6-phosphate dehydrogenase (G6PD) was normal.

Differential count: Neutrophil $32 \%$, lymphocyte $44 \%$, Monocyte $11 \%$, Eosinophil $12 \%$, and basophils $2 \%$. Malaria microscopy investigation was positive for $P$. falciparum (stage: ring form, density:+ and parasite Index: 40.56 per uL). Biochemical parameters were within normal range.

The parasite shown in Figure 1, identified as full-size microfilaria, is Wuchereria bancrofti based on morphological characteristics, with the presence of sheath when stained with Giemsa stain by using standard microscopic method. Cephalic (head) end and tail tapering to delicate point at the tip are visible with morphometric measurements approximately: $260 \pm 15 \times 5.5 \pm 1.5$ $\square \mathrm{m}$ for length and width respectively.

Microfilaria (MF) has a different periodicity related to the presence and biting behavior of the local competent vector, within geographic variation. In our case, it could be hypothesized that the patient was infected with nocturnally subperiodic strain, since MF was present throughout the day with a peak at night; the sample was collected at 9:00 initially in the morning and 21:00 at night after admission. The case was reported to the regional and national surveillance team, since malaria and filariasis are notifiable diseases under the surveillance program of the Ministry of Health Sultanate of Oman. During the interview, the patient declared that she had been treated last year for malaria in her home country. This shows that infections with other blood-borne parasites are common in malaria-endemic areas, as the patient belongs to one of Africa's high-endemic areas and is under MDA WHO treatment and prevention strategy.

\section{Management}

P. falciparum treatment: The patient was treated according to the Oman Ministry of Health standard guidelines with a combination of Artemether $20 \mathrm{mg}$ and Lumefantrine $120 \mathrm{mg}$ ( 4 tablets: $560 \mathrm{mg}$ twice a day for 3 days), followed by Primaquine (45 mg) tablet as a single dose.

Filariasis treatment: Due to non-availability of diethylcarbamazine, the patient was prescribed Albendazole (400mg) and
Ivermectin $(200 \mathrm{ug} / \mathrm{kg})$ for microfilariae as a single dose under Mass Drug Administration WHO strategy (2 drug regimen), followed by Doxycycline $200 \mathrm{mg}$ per day for 6 weeks.

Supportive care: Proper counseling for general hygiene, foot and limb care, application of moisturizing cream and the use of graduated compressed limbs stocks to prevent lymphedema. The patient responded well to the antimalarial and anti-helminthic drugs; peripheral smear was tested after 6 weeks for parasite clearance with no further complaints.

\section{Discussion}

The number of travelers and the expatriate workforce has significantly increased over the last 2 decades in Oman, leading to an increasing number of travel-related infections. These account for the $8 \%$ (558) of all notifiable infections in Oman, according to an epidemiological review from 1999 to 2003 , in which 7022 cases of cholera, chikungunya, dengue, filariasis, leptospirosis, meningococcemia, poliomyelitis, measles, schistosomiasis, A virus hepatitis, typhoid and paratyphoid fever were reported to the National Communicable Department of Surveillance and Control. ${ }^{5}$

Oman is considered non-endemic for Malaria and LF. This is likely to be indirectly related to the strong and strict vector-control policies put in place to maintain the "free-malaria" status of the country, as well as to limit the transmission of the mosquitoborne diseases, which has led to the absence of local occurrence of filarial transmission. Majority of cases were travel-related, imported during travel to endemic regions mainly from South East Asia and African countries. Neglected tropical diseases including vector borne diseases are under surveillance by the Oman Ministry of Health along with other major communicable diseases. In Oman, ongoing surveillance and control measures for all notifiable diseases are established in all province through the Medical Fitness Examination Centers (MFACs) and the Communicable Diseases Surveillance and Control departments.

Chavatte and Jureen published a case study in 2017, which described a case imported in Singapore from India, with asymptomatic Bancroftian Filariasis and coinfection with Plasmodium vivax. Laboratory findings also showed thrombocytopenia. India is considered as one of the endemic countries for LF, where MDA is active under the WHO's treatment and prevention strategy. In this study, the patient responded well to the 2-drug regimen of Albendazole and Ivermectin. The study also highlighted that Singapore remains vulnerable to the introduction of Bancroftian Filariasis, especially due to the large number of foreigners arriving from LF endemic countries, as well as the local travelers and also due to the natural presence of competent local vector. This was classified as an imported relapsing case of malaria and filariasis, which was acquired before the relocation to Singapore. ${ }^{6}$ Our case was also imported from a high endemic country, but filariasis coinfection was found with P. falciparum. This concomitant imported case

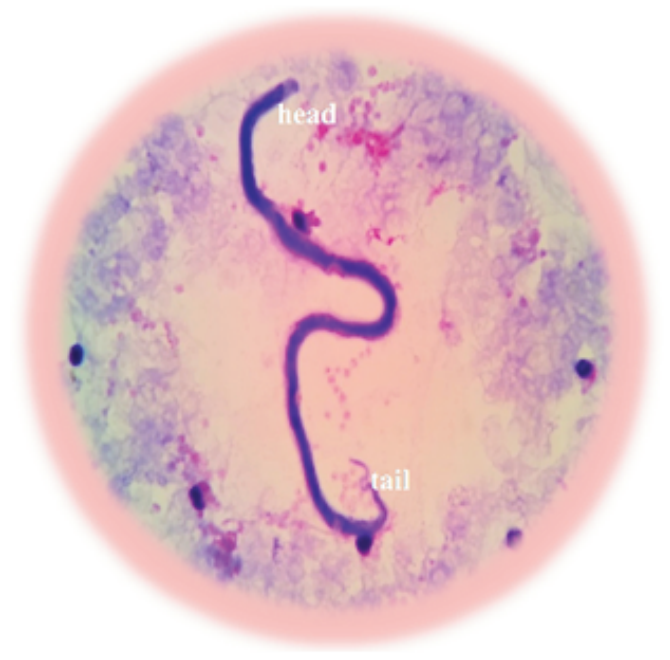


suggests that infections with other bloodborne parasites are common in areas where malaria is endemic, and it appears to be a common feature. Patient was infected with nocturnally subperiodic strain as MF was present throughout the day with a peak at night, which was similar to the case described at Singapore.

Malaria and lymphatic filariasis coinfection is more likely to occur where the prevalence of both diseases is high, which was shown by the study conducted by Muturi et al. in Kenya. ${ }^{7,8}$

Other reports from India in 2018 describe coinfection of filariasis and malaria and with 'unusual crisis' of Plasmodium vivax, which is rarely seen on peripheral smear. The case study of concomitant infection was the second of its kind in India, according to the author's literature review. The case reported that the patient was brought in a state of altered sensorium with complaints of fever and chills for 3 days and hepatosplenomegaly. ${ }^{7}$

Our case highlights incidental concomitant infection of LF with falciparum malaria in a Nigerian woman, who came to Oman for the first time from a high-endemic area. The patient had onset for fever and headache for 2 days before her arrival to Oman, for which she was tested for malaria. Other laboratory parameters including full blood count and biochemistry were within normal range. Also, this could be possibly an imported relapsing case of malaria, since the patient was diagnosed with malaria during the last months of 2018. The filariasis was acquired before her arrival to Oman, because she stayed around 1 month in Buraimi, Oman, and no vector was found near the places where she stayed.

In 2018, LF was only detected at the
Regional Al Buraimi Medical Fitness and Examination Center in a newly arrived Indian citizen to Oman. Therefore, the introduction of such cases of LF from high endemic regions highlighted the importance of early detection and timely treatment of such Neglected Tropical Diseases (NTD). Local transmission can be avoided because possible vectors such as Anopheles subtypes and Culicine mosquitoes including Culex are indigenous in some parts of Oman.

Filariasis is sometimes difficult to diagnose as microscopy is the only diagnostic method available. Depending on the geographical region from where the parasites originates, MF has a different periodicity as it appears in the blood usually at night by using thin/think film microscopy methods.

\section{Conclusions}

There is a need for careful research on co-infection with malaria cases after the arrival of people from their country of origin, especially from areas with high endemicity for helminthic diseases. The documentation of co-infection, its presentation, its progress and treatment differ within WHO regions. There is a general lack of awareness among health workers about LF (classified as NTD by the WHO). LF could pose a potential threat to public health, whether occurring alone or as a co-infection, considering the fact that migration of travelers to and from endemic countries has increased. Therefore, this case report should definitely contribute to raise the awareness of LF, both at local and national level among all health workers.

\section{References}

1. WHO. World Malaria Report 2018. Available from: https://www.who. int/malaria/publications/world-malariareport-2018/ report/en/

2. WHO EMRO. Filariasis. Available from: http://www.emro.who.int/healthtopics/filariasis/index.html

3. WHO. Global Programme to Eliminate Lymphatic Filariasis. Progress Report 2000-2009 and Strategic Plan 20102020. Geneve: WHO; 2010.

4. WHO. Lymphatic Filariasis. Fact sheet 2019. Available from: https://www. who.int/news-room/fact-sheets/detail/ lymphatic-filariasis.

5. Al Abri, SS, Abdel-Hady DM, Al Mahrooqi SS, et al. Epidemiology of travel-associated infections in Oman 1999-2013: A retrospective analysis. Travel Med Infect Dis 2015;13:388-93.

6. MarcCavatte J, Jureen R. Imported Asymptomatic Bancroftian Filariais Discovered from a Plasmodium Vivax Infected Patient: A Case Report from Singapore. Case Rep Infect Dis 2017:8.

7. Shetty JB, Kini S, Phulpagar M, Meenakshi B. Coinfection of malaria and filaria with unusual crisis forms. Trop Parasitol 2018;8:44-6.

8. Muturi EJ, Mbogo CM, Mwangangi JM, et al. Concomitant infections of Plasmodium falciparum and Wuchereria bancrofti on the Kenyan coast. Filaria J 2006;5:8. 